\title{
Benchmark Studies of the Effectiveness of Structural and Internal Materials as Radiation Shielding for the International Space Station
}

\author{
J. Miller ${ }^{1}$, C. Zeitlin ${ }^{1}$, F. A. Cucinotta ${ }^{2}$, L. Heilbronn ${ }^{1}$, D. Stephens ${ }^{3}$ and J. W. Wilson ${ }^{4}$
}

1. Lawrence Berkeley National Laboratory, University of California, Berkeley, CA 94720

2. NASA-Johnson Space Center, Houston, TX 77058

3. Department of Nuclear Engineering, University of Tennessee, Knoxville, TN 37996

4. NASA-Langley Research Center, Hampton, VA 23681

Number of copies submitted: 4

Number of figures: 10

Number of tables: 5

Proposed running head: $\quad$ Effectiveness of ISS Materials as Radiation Shielding

Send correspondence to:

J. Miller

MS 74-197

Lawrence Berkeley National Laboratory

Berkeley, CA 94720

Phone: (510) 486-7130

Fax: (510) 486-7934

E-Mail: miller@lbl.gov

Miller, J., Zeitlin C., Cucinotta, F. A., Heilbronn, L., Stephens, D. and Wilson, J. W.

A Study of the Effectiveness of Space Station Radiation Shielding Materials. Radiat. Res. 


\begin{abstract}
Accelerator-based measurements and model calculations have been used to study the heavy ion radiation transport properties of materials in use on the International Space Station (ISS). Samples of the ISS aluminum outer hull were augmented with various configurations of internal wall material and polyethylene. The materials were bombarded with high energy iron ions characteristic of a significant part of the Galactic Cosmic Ray (GCR) heavy ion spectrum. Transmitted primary ions and charged fragments produced in nuclear collisions in the materials were measured near the beam axis, and a model was used to extrapolate from the data to lower beam energies and to a lighter ion. For the materials and ions studied, at incident particle energies from $1037 \mathrm{MeV} /$ nucleon down to at least $600 \mathrm{MeV} /$ nucleon, nuclear fragmentation reduces the average dose and dose equivalent per incident ion. At energies below 400 $\mathrm{MeV} /$ nucleon, the calculation predicts that as material is added, increased ionization energy loss produces increases in some dosimetric quantities. These limited results suggest that the addition of modest amounts of polyethylene or similar material to the interior of the ISS will reduce the dose to ISS crews from space radiation; however the radiation transport properties of ISS materials should be evaluated with a realistic space radiation field.
\end{abstract}




\section{Submitted to Radiation Research}

\section{Introduction}

Crews on the International Space Station (ISS) are spending much more time in space than have all but a small number of their astronaut and cosmonaut predecessors. Management of radiation risk for these individuals requires accurate determination of the local radiation field at various locations within the ISS for various mission conditions and, where feasible, measures to reduce the radiation dose (1).

Recent data from the Mir space station $(2,3)$ and from Space Shuttle flights at high orbital inclination $(4,5,6)$ relative to the equator show that more than $50 \%$ of the dose equivalent incurred by ISS crew (except during solar particle events) will be from high energy nuclei $(\mathrm{Z} \geq 1)$ in the galactic cosmic radiation (GCR), and of that amount a substantial fraction will be from highly charged and energetic heavy nuclei $(\mathrm{HZE})(\mathrm{Z}>2)$. The large contribution of these particles to the dose equivalent is due to the high orbital inclination, $51.6^{\circ}$, of the ISS. The geomagnetic field strength diminishes near the poles, allowing HZE particles which are deflected at lower latitudes to penetrate to objects in low Earth orbit. The inclination for most Space Shuttle flights prior to the start of ISS operations was $28.5^{\circ}$, where most of the radiation dose is from trapped protons in the South Atlantic Anomaly (7).

GCR HZE particles are most abundant at energies between a few tens and several hundred $\mathrm{MeV} /$ nucleon, with the abundance falling off approximately exponentially above 1 GeV/nucleon(8). The GCR flux is dominated by protons, but since ionization energy loss, and therefore absorbed dose, is proportional to the square of the particle charge, ions as heavy as iron (charge, $Z=28$ ), may be biologically significant. Most GCR HZE particles are sufficiently energetic to penetrate to many locations within the ISS. Ionization energy loss also slows incident particles, making them more densely ionizing and further increasing the dose delivered along the trajectories of particles that do not stop. Nuclear fragmentation of the incident GCR ions in the ISS hull and interior materials changes the incident radiation field, in most cases 


\section{Submitted to Radiation Research}

decreasing the dose per incident particle but adding to the complexity of the radiation field inside the ISS.

One way of reducing radiation dose is by the addition of shielding at locations within the ISS, such as sleep stations, where crewmembers spend large amounts of time. In-flight measurements and accompanying model calculations $(2,3,5)$ have shown polyethylene to be superior to aluminum for decreasing both the absorbed dose and the dose equivalent. This is consistent with biological (2) and physical $(9,10)$ model calculations which point to the advantages of hydrogenous materials_ — and the possible deleterious effects of high-Z materialsas radiation shielding.

The two principal interactions for charged particles in matter are excitation and ionization due to electromagnetic interactions with orbital electrons of the target atoms, which slow down incident particles (and stop some of them), and nuclear interactions, which break the incident heavy ions into lighter fragments ${ }^{1}$. To first order, for a given areal density and a given incident particle, ionization energy loss increases with the charge-to-mass ratio of the target nucleus, $Z / A$, so that hydrogen is highly effective compared to other materials ${ }^{2}$. Furthermore, because the nuclear interaction cross sections are approximately proportional to $\left(A_{t}^{1 / 3}+A_{p}{ }^{1 / 3}\right)^{2}$, where $A_{t}$ and $A_{p}$ are the target and projectile mass numbers, there is more fragmentation of the incident particle per unit mass of shielding in hydrogen than in other materials. Thus by mass, hydrogen is the most efficient material for shielding against heavy ions.

The situation in spaceflight is more complicated than, for example, shielding at a nuclear reactor or particle accelerator, because practical shielding thicknesses are insufficient to stop most of the incident GCR particles. For example, $500 \mathrm{MeV} /$ nucleon iron ions have a range of $4.5 \mathrm{~cm}$ in aluminum and over $10 \mathrm{~cm}$ in polyethylene. For $500 \mathrm{MeV} /$ nucleon alpha particles the corresponding ranges are $55 \mathrm{~cm}$ and $122 \mathrm{~cm}$. For trapped electrons and protons, and possible solar particle events, the particles are of lower energies, and thus more densely ionizing than 


\section{Submitted to Radiation Research}

their counterparts in the GCR, and the addition of shielding has someadvantages, although it does increase the dose per particle for particles that do not stop. For the GCR, nuclear interactions further complicate matters, because the more lightly charged fragments have longer ranges than the primary. Thus the radiation dose from heavy ions inside the spacecraft will be a sum of competing effects: fragmentation, which tends to decrease the dose, and ionization, which tends to increase the dose for particles that do not stop before reaching the crewmember's body. This tradeoff is the source of the striking result $(2,7)$ that for realistic spacecraft shielding thicknesses, the addition of some materials actually increases the dose equivalent from GCR HZE particles over the unshielded value.

Previous shielding studies were done either in flight at specific locations on the Mir space station or the Space Shuttle, or using model calculations. The measurements had the advantage of being made in space, but the disadvantages of relatively low statistics and limitations on location of the experiments and resolution of the instrumentation. The purpose of the present study was to make a high statistics measurement with a single ion-energy combination and several material combinations under consideration for use on the ISS, in particular in the crew sleeping quarters. Recognizing the limitations of measurements with one ion species at one energy, the data were used to evaluate the accuracy of a model similar to those used in previous studies, and the model was then used to extrapolate from the data.

\section{Materials and Methods}

The measurements were made at the Brookhaven National Laboratory Alternating Gradient Synchrotron (BNL AGS) with beams of ${ }^{56} \mathrm{Fe}$ ions. ${ }^{56} \mathrm{Fe}$ is the heaviest ion present in significant numbers in the GCR. The energy at extraction from the AGS was 1087 $\mathrm{MeV} /$ nucleon; after passing through upstream beamline elements and detectors the beam energy at the entrance to the target was $1037 \mathrm{MeV} /$ nucleon. 


\section{Submitted to Radiation Research}

\section{Detectors and Data Acquisition}

The detector configuration for this experiment is shown schematically in Fig. 1. The experimental setup was similar to those for previous measurements by our group $(11,12,13,14)$ using silicon detectors, in this case a series of detectors of thickness $300 \mu \mathrm{m}$ ("TR"), $3 \mathrm{~mm}$ (d3mmU and $\mathrm{d} 3 \mathrm{~mm} 1-4)$ and $1 \mathrm{~mm}$ position-sensitive(PSD1 and PSD2). A charged particle passing through the silicon liberates one electron-hole pair per $3.6 \mathrm{eV}$ energy deposited. The resulting signal is amplified, digitized, and stored for offline analysis.

The detectors upstream of the target were used to identify Fe beam particles by their energy deposition in the silicon. The three pairs of detectors downstream of the target were used to determine fragment charges and energies. They subtended angles of $7.5^{\circ}$ (PSD2), $2.5^{\circ}$ $(\mathrm{d} 3 \mathrm{~mm} 1 / 2)$ and $1.0^{\circ}(\mathrm{d} 3 \mathrm{~mm} 3 / 4)$ centered around the beam axis. Data acquisition was initiated by an event trigger, defined as a coincidence of beam signals in detectors TR and $\mathrm{d} 3 \mathrm{mmU}$. The discriminator thresholds were set to accept as many Fe beam events as possible, and therefore some triggers were generated by fragments within a few charge units of Fe created by upstream nuclear interactions. These events were eliminated in the offline analysis, as were events where two or more Fe ions passed through the system close enough in time to be recorded in a single event.

For each event, the data acquisition system recorded energy deposition in each detector, position information from PSD1 and PSD2 and additional high gain energy deposition signals from each of the four downstream detectors (d3mm1-4). The high gain signals were used to help identify the lightest charged fragments. Each PSD consisted of a pair of $1 \mathrm{~mm}$-thick detectors measuring vertical and horizontal position in the plane normal to the beam axis. Each of these detectors generated three signals: two position-dependent ("L" and "R" or " $U$ " and "D") and one total energy loss ("DEX" or "DEY"). For example, the output signals from the horizontal detector of PSD1 were DEX1, L1 and R1. The position-dependent signals can be used to obtain 


\section{Submitted to Radiation Research}

the particle coordinates normal to the beam, but in this analysis they were used only to select on particle energy loss.

\section{Target Materials}

The targets, provided by NASA-Langley Research Center and NASA-Johnson Space Center, were ISS structural and internal materials and sheets of polyethylene $\left(\mathrm{CH}_{2}\right)$. The ISS materials were samples of the aluminum hull and the crew quarter (CQ) interior wall, which is composed of noise abatement and fire retardant materials ${ }^{3}$. Specifications of these materials are in Table 1. The polyethylene was in two forms: an "encapsulated PE" consisting of $12 \mathrm{~mm}$ polyethylene sandwiched between sheets of $1 \mathrm{~mm}$ graphite-epoxy composite; and a "PEaugmented CQ wall" consisting of $12 \mathrm{~mm}$ polyethylene sewn into a CQ wall sample. These were combined in several different configurations, as discussed in the Results, below. Data were also taken with no target in order to measure "background" production of fragments and losses of primaries, for example by interactions in the detectors.

\section{Data analysis}

The analysis procedure was similar to that described in detail in refs. (11-14). Histograms and scatterplots were made of the energy deposition, $\Delta \mathrm{E}$, in the detectors, and graphical cuts were used to select or reject events according to specific criteria. The first set of cuts, on detectors upstream of the target, required a single iron ion in both TR and $\mathrm{d} 3 \mathrm{mmU}$ and correlated $\Delta \mathrm{E}$ signals consistent with an iron ion in the PSD1 detectors. For events satisfying these cuts, subsequent cuts selected events with either a surviving primary ion or one or more fragments. The sample of good events was defined by requiring correlated energy loss in the detectors of each pair. An additional set of cuts excluded events where a beam ion interacted in one of the detectors downstream of the target. Fluence spectra were obtained from the observed fragment spectra in a series of steps: 


\section{Raw counts}

The detectors downstream of the target subtended small angles around the beam axis, and thus for most events recorded a single primary ion or a small number of fragments at or near the beam velocity. There arepeaks in the $\Delta \mathrm{E}$ spectra corresponding in most cases tothe individual fragment charges, as shown in Fig. 2. The number of fragments in each peak was determined by summing over the bins between valleys. Double Gaussian fits to adjacent peaks were used to allocate the relatively small number of counts in the regions where two peaks overlapped significantly.

\section{Fragment charge distributions}

The fragment $\Delta \mathrm{E}$ spectrum in Fig. 2 can be converted to a charge distribution by making use of the fact that when all particles are at or near the same velocity the energy loss of charged particles in this energy range is approximately linear in $Z^{2}$. The spectra in Fig. 3 were obtained by assigning $Z=26$ to the iron peak and scaling the other data points by the ratio of the deposited energy to the peak iron energy. The iron peaks have been excluded from these histograms in order to emphasize the differing resolution of fragment species in the three detector pairs.

Figure 3 shows that the particle charge resolution improves with distance from the target. The PSD2 detectors, closest to the target and with the largest diameter, have the poorest resolution, partly because the PSD's are thin, but mainly due to their relatively large angular acceptance. A large-acceptance detector will see a higher fragment multiplicity than a smallacceptance detector on the same event. Events with no leading fragment with $\mathrm{Z}$ greater than about half the beam charge can produce many combinations that yield effective Z's at noninteger values, obscuring single-fragment peaks in the lower half of the charge spectrum. This is true especially for the smallest charges, where multiple fragments are more likely. The detector acceptance decreases with increasing downstream distance, and because fragments have some transverse ${ }^{4}$ momentum relative to the beam direction, they are more likely to miss the 


\section{Submitted to Radiation Research}

downstream detectors, reducing the likelihood of multiple particles hitting those detectors. Thus the detector pair farthest downstream, $\mathrm{d} 3 \mathrm{~mm} 3 / 4$, has the sharpest charge peaks and the highest yield of $Z=1$ and 2 fragments. Note also that the peak at $Z=0$, which contains events in which no charged fragment passed through the detectors, increases with decreasing acceptance, as is to be expected.

To improve the resolution for smaller charges, the signals for $\mathrm{d} 3 \mathrm{~mm} 1-4$ were split and, on one branch, amplified further. Figure 3d shows the "high gain" spectrum corresponding to the low end of Fig. 3b. This spectrum illustrates, albeit with poor statistics, the effect of multiple fragments. Non-integral charge peaks are in many cases due to fragments from the same interaction that are closely correlated in phase space. For example the peak between $\mathrm{Z}=3$ and $\mathrm{Z}=4$ is produced by three He fragments. This final state gives an effective charge $\mathrm{Z}_{\mathrm{eff}}=$ $\sqrt{2^{2}+2^{2}+2^{2}}=3.46$. Simultaneous fits of multiple Gaussians reveal that most of the other light charge peaks, especially $\mathrm{Z}=1$ and $\mathrm{Z}=2$ are broadened by additional peaks $(e . g \cdot p+p, p+p+p, \alpha+p$, $\alpha+p+p$ ) for which the effective charges cannot be resolved from the neighboring integral charge peaks. In this analysis, we count only the heaviest particle. For example, for purposes of calculating the fluence, the $\alpha+p+p$ final state is recorded as a single particle with $\mathrm{Z}=2$. A more detailed discussion of events with multiple light fragments detected in coincidence can be found in Ref. (13).

\section{Corrections}

Corrections were applied for background due to beam and fragment interactions in materials other than the target. Interactions in air gaps, detector dead layers, etc., were accounted for by subtracting, for each fragment species, the fluence in the target-out run; interactions in the silicon stack were corrected for by normalizing to the expected fraction of ions surviving as a function of charge and cumulative thickness of silicon. ( $2 \mathrm{~mm}, 8 \mathrm{~mm}$ and $14 \mathrm{~mm}$ for PSD2, 


\section{Submitted to Radiation Research}

$\mathrm{d} 3 \mathrm{~mm} 1 / 2$ and $\mathrm{d} 3 \mathrm{~mm} 3 / 4$ respectively.) The survival fraction was calculated using the geometric cross section, assuming the atomic number of the most common naturally-occurring isotope of each element. (No attempt was made to take isotopic production cross sections into account here, but this is a small correction. ${ }^{5}$ )

\section{Fluence}

The corrected fluence ${ }^{6}$ for each $\mathrm{Z}, \phi^{\prime}(\mathrm{Z})$, measured in a given downstream detector pair is calculated from the observed number of counts $\mathrm{N}(\mathrm{Z})$ as follows:

Apply a correction for the observed number of fragments for interactions in the silicon detectors:

$$
\mathrm{N}^{\prime}(Z)=\mathrm{N}(\mathrm{Z}) / \mathrm{P}\left(\mathrm{Z}, \Delta \mathrm{t}_{\mathrm{Si}}\right)
$$

where $\mathrm{P}\left(\mathrm{Z}, \Delta \mathrm{t}_{\mathrm{Si}}\right)$ is the surviving fraction for a fragment with charge $\mathrm{Z}$ after passing through detectors including this pair

Calculate the fluence

$$
\phi(Z)=N^{\prime}(Z) / \Sigma_{Z} N^{\prime}(Z)
$$

Apply a correction for interactions in the air gaps, etc., as determined with no target present:

For the beam ions:

$$
\begin{aligned}
& \phi^{\prime}(26)=\phi(26) / \phi(26)_{\text {target out }} \\
& \delta \phi^{\prime}(26)=\left[\phi(26)(1-\phi(26)) / \Sigma_{\mathrm{Z}} \mathrm{N}(\mathrm{Z})\right]^{1 / 2}
\end{aligned}
$$

and for fragments:

$$
\begin{aligned}
& \phi^{\prime}(\mathrm{Z})=\phi(\mathrm{Z})-\phi(\mathrm{Z})_{\text {target out }} \cdot \phi^{\prime}(26) \\
& \delta \phi^{\prime}(\mathrm{Z})=\left[\delta \phi^{2}(\mathrm{Z})+\phi^{\prime 2}(26) \cdot \delta \phi^{2}(\mathrm{Z})_{\text {target out }}+\delta \phi^{\prime 2}(26) \cdot \phi^{2}(\mathrm{Z})_{\text {target out }}\right]^{1 / 2}
\end{aligned}
$$

The uncertainty in the transmitted iron fluence, $\phi^{\prime}(26)$, reflects the fact that the parent distribution is binomial: a beam ion either fragments or does not. 


\section{Results and Discussion}

Five combinations of target materials were exposed to the beam, and data were also taken with no target. The targets were:

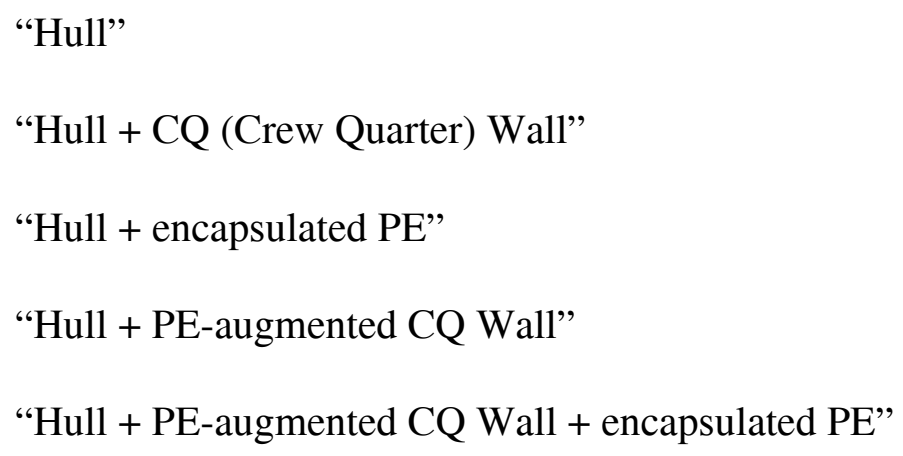

The heavy ion component of the space radiation environment is unique in that the dose rate is low, but the dose per particle can be quite high. Determination of the relative importance of biological effects produced by accumulated dose compared to the effects of single particles is outside the scope of this paper, but either or both may prove to be significant (15), and so we have considered the data in both contexts.

\section{Fragment and LET spectra}

For very energetic ions, which lose little energy in the targets, breaking the incident particles into more lightly ionizing fragments reduces the total dose per incident ion. Figure 4 is a plot of the fluence (per incident iron ion) of the surviving primary ions and each fragment charge produced in each target as recorded in the detector pair furthest downstream $(\mathrm{d} 3 \mathrm{~mm} 3 / 4)$. The likelihood of fragmentation increases as material is added inside the ISS hull: approximately $60 \%$ of the beam ions remain intact after passing through the thickest target, compared to $93 \%$ after passing through only the $7 \mathrm{~mm}$ aluminum outer hull.

The more relevant quantity from a radiation protection standpoint is the energy deposited by the charged particles. Figure 5 shows the primary ion and fragment fluences in Fig. 4 as a 


\section{Submitted to Radiation Research}

function of calculated LET in water. The abscissa is the LET for each charge state in Fig. 4.

Here we define LET to be the $\mathrm{LET}_{\infty}$, obtained by scaling the calculated $\mathrm{LET}_{\infty}$ of the Fe beam in water by $\mathrm{Z}^{2}$ for each fragment. ${ }^{7}$ This plot is similar to Fig. 4, but shows both the slight increase in LET for each charge (including the beam) with increasing material thickness, and the nonlinearity of the relation between LET and charge. This increase is more than offset by the decreased fluence of the high-LET primary ions (as can be seen also in the track-average LET $^{8}$, below).

\section{Angle-dependence}

We detected only particles emitted at small angles with respect to the beam axis; comparing the data from the three detector pairs gives some insight into the angular distribution of the produced particles, and thus of the dose. As can be seen from Table 2, the track-average LET, $\langle\mathrm{L}\rangle_{\text {trk }}$, does not decrease in proportion to the angular acceptance. For example, the acceptance of PSD2 is almost a factor of eight greater than that of $\mathrm{d} 3 \mathrm{~mm} 3 / 4$, yet for the thickest target, which shows the largest decrease in $\langle\mathrm{L}\rangle_{\text {trk }}$ between the two detector pairs, the trackaverage LET calculated from the $\mathrm{d} 3 \mathrm{~mm} 3 / 4$ data is only $9 \%$ less than that calculated from the PSD2 data. Also, although the detection system could not in most cases resolve multiple particles from the same event, the distinct peaks in the $\mathrm{d} 3 \mathrm{~mm} 3 / 4$ spectra over the ranges of observed charge and LET indicate that most interactions produce only a single particle within the small acceptance of this detector pair.

These data are consistent with the expectation that the produced fragments will be concentrated within a narrow cone around the beam axis due to their relatively small momentum transverse to the beam ${ }^{9}$. This has implications for the dose, as discussed below

3. Dose

The dose per incident iron ion is given by:

$$
\mathrm{D}=1.602 \sum_{\mathrm{i}} \mathrm{L}_{\mathrm{i}} \phi_{\mathrm{i}}
$$




\section{Submitted to Radiation Research}

where $\mathrm{D}$ is in $\mathrm{nGy}, \mathrm{L}_{i}$ is the average LET in $\mathrm{keV} / \mu \mathrm{m}$ of the $\mathrm{i}^{\text {th }}$ species in the $\mathrm{Z}$ histogram, $\phi_{i}$ is the fluence (in particles- $\mathrm{cm}^{-2}$ ) of the $\mathrm{i}^{\text {th }}$ species, and the summation is over all charges. We have chosen our normalization so that the sum of the $\phi_{\mathrm{i}}$ is exactly 1 . Figure 6 shows a histogram of $\mathrm{L}_{\mathrm{i}} \cdot \phi_{\mathrm{i}}$ as a function of $\mathrm{Z}$ for each of the ISS materials. Each point represents the contribution to dose of that particular ion species. Since dose is proportional to $\mathrm{Z}^{2}$, the lightly charged fragments contribute relatively little to the dose compared to higher $\mathrm{Z}$ fragments. The dose equivalent falls even faster with decreasing fragment charge, due to the sharp fall-off of the ICRP60 quality factor, Q, with decreasing LET below $100 \mathrm{keV} / \mu \mathrm{m}$. Summing the data in Fig. 6 over all charges gives $\langle\mathrm{L}\rangle_{\text {trk }}$. If we assume, as is supported by the data, that for most interactions the dose to a small volume along the beam axis is carried by a single fragment, then the average dose per incident iron ion to that small volume is proportional to the track-average LET. Similarly, the dose equivalent per incident iron ion is given by:

$$
\mathrm{H}=\mathrm{DQ}=\mathrm{D} \times \frac{1}{\mathrm{D}} \int \mathrm{Q}(\mathrm{L}) \mathrm{D}(\mathrm{L}) \mathrm{dL}=\sum_{\mathrm{i}} \mathrm{Q}\left(\mathrm{L}_{\mathrm{i}}\right) \mathrm{L}_{\mathrm{i}} \phi_{\mathrm{i}}
$$

where $\mathrm{H}$ is in $\mathrm{nSv}$ and the other quantities are as before. Figure 7 shows a histogram of the quantity $\mathrm{Q}\left(\mathrm{L}_{\mathrm{i}}\right) \cdot \mathrm{L}_{\mathrm{i}} \cdot \phi_{\mathrm{i}}$ vs. Z, analogous to that in Fig. 6 but with the additional weighting by $\mathrm{Q}$. The contribution from light fragments relative to that of the surviving iron is roughly five orders of magnitude smaller. This is in contrast to Fig. 6, where the light-fragment contributions are about three orders of magnitude lower than that of the primary.

In view of the fact that the actual charged fragment multiplicity is, by charge conservation, always greater than one, one must take into account the size of the biological volume of interest. For example, for applications to sites with volumes on the order of a single cell, the assumption that all the dose is carried by a single forward-going particle is a good one; but for calculating, e.g., skin dose, fragments produced at larger angles should be considered, and the production of multiple fragments taken into account. Particles emitted at large angles also tend to be slower 


\section{Submitted to Radiation Research}

than those along the beam axis, and so the dose per particle may be larger than that of forwardgoing particles.

Table 3 shows the ratios of $\langle\mathrm{L}\rangle_{\text {trk }},\langle\mathrm{Q}\rangle_{\text {trk }}$ and $\langle\mathrm{L} \cdot \mathrm{Q}\rangle_{\text {trk }}$ for the various configurations of the 7 mm aluminum ISS hull and other materials, to the values for the hull alone, calculated using the data from detector pair $\mathrm{d} 3 \mathrm{~mm} 3 / 4$. All three quantities are observed to decrease with increasing material thickness, reflecting the increased fragmentation. We note that the quantity $L \cdot Q$ for a given fragment is proportional to the "conventional risk cross section", ${ }_{r} \sigma_{Z}(\mathrm{~L})$, the probability of excess cancer mortality per unit fluence, introduced by Curtis, et al. (16). ${ }^{10}$

Ionization energy loss somewhat counteracts the effect of nuclear fragmentation in reducing dose: the dose (and in most cases the dose equivalent) for any given charge increases with increasing material thickness, due to ionization energy loss. In Ref. (11), it was shown that that with a $510 \mathrm{MeV} /$ nucleon ${ }^{56} \mathrm{Fe}$ beam incident on polyethylene targets, the competing effects balanced and track-averaged LET was nearly constant for three depths $(0 \mathrm{~cm}, 2 \mathrm{~cm}$, and $5 \mathrm{~cm})$ of shielding. However, with the higher beam energy in the present experiment, the reduction in dose due to fragmentation of the primary iron ions is the dominant effect. Figure 8 shows the attenuation of the primary iron ions vs. the track-average LET for each material. While a fragment with a given charge may, depending upon where in the target it is produced, deposit more energy when produced in a thicker target, the average energy deposition per incident ion decreases with increased material thickness for the materials studied. This applies both to dose equivalent accumulated over extended periods and to energy deposition by a single particle at these energies.

For long exposure times, the dose-average (as opposed to track-average) LET and quality factor are important measures of radiation risk. We have found for every material combination that at $1037 \mathrm{MeV} /$ nucleon incident energy the dose-average LET is within 5\% of the beam LET 


\section{Submitted to Radiation Research}

of 147.4, reflecting the fact that even with as many as $40 \%$ of the primaries fragmenting in the shielding materials, the dose-average quantities are dominated by contributions from the surviving primary ions. This has been noted in previous measurements with the radiobiology heavy ion beam at the AGS (10). Similarly, in no case is the dose-average quality factor decreased by more than $2 \%$ from the nominal value of 24.7 for $1000 \mathrm{MeV} /$ nucleon iron ions, obtained using the ICRP60 Q vs. LET relationship.

\section{Model comparison and beam energy dependence}

The first two columns in Table 4 compare the dose- and track-average LET and quality factor calculated from the data to results of a Monte Carlo model similar to the one described in Ref. (17). The target is the ISS hull material ( $7 \mathrm{~mm} \mathrm{Al})$ with polyethylene augmentation (1 mm graphite-epoxy $+12 \mathrm{~mm}$ polyethylene $+1 \mathrm{~mm}$ graphite-epoxy.) The Monte Carlo uses cross sections generated by the NUCFRAG2 fragmentation code (18). The model reproduces the data well. The three right-most columns were calculated using the model for iron ions at 600,400 and $300 \mathrm{MeV} /$ nucleon incident on the same target materials. These energies are close to the peak of the GCR iron ion energy spectrum. The model has also been shown to be in good agreement with data for iron ions at $510 \mathrm{MeV} /$ nucleon (14). ${ }^{1}$. The model calculations give some insight into the contribution of lower energy iron ions to the radiation field inside the ISS.

The ratio of track-average to dose-average LET is about the same at all four energies, which suggests that fragmentation contributes proportionately about the same amount at each energy. At the lower energies, the increase in LET is due primarily to increased ionization energy loss at the lower beam velocity, and the sharp increase in LET at $300 \mathrm{MeV} / \mathrm{nucleon}$ reflects the steep rise in the Bragg curve at low energy. The average energy loss per particle is much greater at $300 \mathrm{MeV} /$ nucleon, but the higher LET results in a decrease in average quality factor, and both effects are enhanced by the addition of material. Table 5 shows, for iron ions at 


\section{Submitted to Radiation Research}

four different incident energies, the calculated track-average LET for aluminum and aluminum augmented with polyethylene. At $300 \mathrm{MeV} /$ nucleon incident energy, the additional polyethylene increases the track-average LET, rather than decreasing it, as is the case at the higher energies. This is once again a consequence of the low-energy behavior of the ionization energy loss. The dose-average LET increases to a similar degree, while the quality factor decreases somewhat, since the ICRP60 quality factor falls with increasing LET above $100 \mathrm{keV} / \mu \mathrm{m}$.

Figures 9 and 10 illustrate the effect of polyethylene as a function of material thickness for iron and silicon ions, where silicon $(\mathrm{Z}=14, \mathrm{~A}=28)$ was chosen because it is much lighter than iron but still heavy enough so that fragmentation might be expected to be a factor. The Monte Carlo code was used to calculate the track average LET and Q-weighted LET at several beam energies spanning the peak of the GCR spectrum for the $7 \mathrm{~mm}$ aluminum ISS hull alone and for the hull augmented by 1,2, 5 and $10 \mathrm{~cm}$ polyethylene. For both iron and silicon, at 600 and $1000 \mathrm{MeV} /$ nucleon, modest decreases in both $\langle\mathrm{L}\rangle_{\text {trk }}$ and $\langle\mathrm{L} \cdot \mathrm{Q}\rangle_{\mathrm{trk}}$ are observed for at least $2 \mathrm{~cm}$ polyethylene. At $400 \mathrm{MeV} /$ nucleon, the results are mixed, and at lower energies, adding even 1 cm polyethylene increases both $\langle\mathrm{L}\rangle_{\text {trk }}$ and $\langle\mathrm{L} \cdot \mathrm{Q}\rangle_{\text {trk }}$. Below $200 \mathrm{MeV} /$ nucleon, and at higher energies for some thicknesses of polyethylene, the incident beams stop in the aluminum, and the residual dose is very small. These calculations, while not exhaustive, indicate that $1-2 \mathrm{~cm}$ is a reasonable thickness of polyethylene augmentation. 


\section{Submitted to Radiation Research}

\section{Conclusions}

We have made an accelerator-based study of the effectiveness of different combinations of materials in use or considered for use on the ISS at attenuating and fragmenting 1037 $\mathrm{MeV} /$ nucleon iron ions. Samples of the aluminum ISS outer hull were augmented variously with crew quarter wall material and polyethylene in several configurations. Polyethylene was chosen for its known advantages in shielding against high energy heavy charged particles. The projectile and energy are characteristic of the trailing edge of the peak of the GCR heavy ion spectrum. Transmitted primary ions and produced charged fragments were measured near the beam axis. The experimental data were used to benchmark a Monte Carlo fragmentation model, and the model was used to extrapolate from the data to lower beam energies.

For the high-energy heavy ions in the experiment, the dose averaged over many incident particles is dominated by the surviving primary ions, and therefore the dose-average LET and quality factor are relatively insensitive to the addition of material in the amounts studied here. However several dosimetric quantities decrease as material is added inside the ISS hull. These include the track-average LET and quality factor and the average dose and dose equivalent per incident primary ion. These results reflect the interplay of nuclear fragmentation and ionization energy loss. Ionization energy loss, and therefore dose, is proportional to the square of the fragment charge and inversely proportional to its velocity. At this energy and for these material thicknesses, energy loss is modest, fragmentation dominates, and the net result is to reduce the average dose per incident ion. Moreover, analysis of the angular distribution of the produced particles shows that the dose in all cases remains concentrated in the forward direction. Thus it is advantageous to add material to fragment the incident ions, even at the expense of slowing the fragments and surviving primaries and making them somewhat more ionizing. 


\section{Submitted to Radiation Research}

The Monte Carlo transport code was used to calculate some of the same quantities for iron and silicon ions at several beam energies spanning the peak of the GCR iron spectrum. At 600 $\mathrm{MeV} /$ nucleon incident energy there is a modest advantage to adding up to $2 \mathrm{~cm}$ polyethylene inside the ISS hull. However below $400 \mathrm{MeV} /$ nucleon the track-average and dose-average LET and track-average Q-weighted LET increase as material is added.

These limited results suggest that the addition of modest amounts of polyethylene or similar material to the interior of the ISS will reduce the radiation dose to crewmembers from GCR heavy ions with energies near the peak of the GCR energy spectrum. However, given the inhomogeneities in the ISS structure and the complex nature of the radiation field in low Earth orbit, the radiation transport properties of ISS materials and the predictive power of transport models need to be evaluated using a broader sample of the entire flux of GCR and trapped particles, including dosimetric measurements on board the ISS with prototype shielding in place.

\section{Acknowledgements}

We thank P. Saganti (NASA-Johnson Space Center) and M.Y. Kim (NASA-Langley Research Center) for providing the ISS material samples and details of their composition, and the outstanding operations staff of the BNL AGS for providing the iron beams for the experiments. This work was supported by the National Aeronautics and Space Administration-Johnson Space Center under Award No. T5606W and the NASA Space Radiation Health Program under Grant No. L14230C, through the U.S. Department of Energy under Contract No. DEAC03076SF00098. 


\section{Footnotes}

1. Here we consider only projectile fragments, as target fragments will in most cases be absorbed in the shielding material.

2. Ionization energy loss depends on other variables as well, including incident particle velocity and the ionization energy of the target atoms, but hydrogen has the greatest stopping power per unit mass since its $\mathrm{Z} / \mathrm{A}$ is 1 , compared to $\mathrm{Z} / \mathrm{A} \leq 1 / 2$ for virtually every other element.

3. The crew quarter wall consists of three layers: Outer-Nomex flame retardant, Bisco silicone noise abatement layer, Durette flame retardant batting, Bisco and Nomex (five layers); Middle-Nomex honeycomb; Inner-Nomex, Durette and Nomex (three sub-layers). The thicknesses in Table I are totals for each material.

4. It is well-established that fragment transverse momentum increases as fragment mass decreases. See Ref. (14) and references therein.

5. The percentage correction is on the order of $\Sigma_{1} x_{i}\left(\mathrm{~A}+n_{i}\right)^{2 / 3} / \mathrm{A}$ where $x_{i}$, the percentage of the $i^{\text {th }}$ isotope and $n_{i}$, the deviation from the naturally-occurring atomic number, $\mathrm{A}$, are small numbers, and the $n_{i}$ can be positive or negative.

6. For the purposes of this measurement we define the fluence to be normalized to a single incident beam particle, with the transmitted beam or produced fragments emerging from a circle with area $1 \mathrm{~cm}^{2}$ on the downstream face of the target, centered on the beam axis. (See the Appendix to Ref. (11).)

7. This assumes that all measured fragments are at the beam velocity and that LET is linear in $\mathrm{Z}^{2}$, which for relativistic velocities and fragments measured near the beam axis are good approximations, and adequate for determining the relative fragmentation properties of the ISS materials. Exact calculation of LET in water from measured energy loss in silicon requires an independent measurement of the particle velocity, which was not made in this experiment. 
8. Track-average and dose-average LET are calculated as follows:

$$
\begin{aligned}
& \langle\mathrm{L}\rangle_{\text {trk }}=\frac{\sum_{\mathrm{i}} \mathrm{N}_{\mathrm{i}} \cdot \mathrm{L}_{\mathrm{i}}}{\sum_{\mathrm{i}} \mathrm{N}_{\mathrm{i}}} \\
& \langle\mathrm{L}\rangle_{\text {dose }}=\frac{\sum_{\mathrm{i}} \mathrm{N}_{\mathrm{i}} \cdot \mathrm{L}_{\mathrm{i}} \cdot \mathrm{L}_{\mathrm{i}}}{\sum_{\mathrm{i}} \mathrm{N}_{\mathrm{i}} \cdot \mathrm{L}_{\mathrm{i}}}
\end{aligned}
$$

where $N_{i}$ is the number of fragments of the $i^{\text {th }}$ fragment species in the $\mathrm{Z}$ histogram, and $\mathrm{L}_{\mathrm{i}}$ is the LET $_{\infty}$ for that species, calculated as described in the text.

9. Transverse momenta per nucleon are on the order of $100 \mathrm{MeV} / \mathrm{c}$, and longitudinal momenta are around $1700 \mathrm{MeV} / \mathrm{c}$.

10. Calculation of the ${ }_{\mathrm{r}} \sigma_{\mathrm{Z}}(\mathrm{L})$ requires knowledge of the risk coefficients, or the functional dependence of ${ }_{\mathrm{r}} \sigma_{\mathrm{Z}}(\mathrm{L})$ on $\mathrm{Z}$ and $\mathrm{L}$ (13). 


\section{References}

1. NCRP, Radiation Protection Guidance for Activities in Low Earth Orbit. Report 132, National Council on Radiation Protection and Measurements, Bethesda, MD, 2000.

2. G.D. Badhwar, A. Konradi, W. Atwell, M.J. Golightly, F.A. Cucinotta, J.W. Wilson, V.M. Petrov, I.V. Tchernykh, V.A. Shurshakov and A.P. Lobakov, Measurements of the linear energy transfer spectra on the Mir orbital station and comparison with radiation transport models. Radiat. Meas. 26(2), 147-158 (1996).

3. F.A. Cucinotta, J. W. Wilson, J.R. Williams and J.F. Dicello, Analysis of Mir-18 results for physical and biological dosimetry: radiation shielding effectiveness in LEO. Radiat. Meas. 32, 181-191 (2000).

4. G.D. Badhwar and F.A. Cucinotta, Depth dependence of absorbed dose, dose equivalent and linear energy transfer spectra of galactic and trapped particles in polyethylene and comparisons of calculations with models. Radiat. Res. 149, 209-218 (1998).

5. T. Sakaguchi, T. Doke, N. Hasebe, T, Hayashi, T. Kashiwagi, J. Kikuchi, S. Kono, S. Nagaoka, T. Nakano, T. Takagi, K. Takahashi and S. Takahashi, LET distribution measurement with a new real-time radiation monitoring device-III onboard the Space Shuttle STS-84. Nucl. Instr. and Meth. In Phys. Res. A 437, 75-87 (1999).

6. G.D. Badhwar and F.A. Cucinotta, A comparison of depth dependence of dose and linear energy transfer spectra in aluminum and polyethylene. Radiat. Res. 153, 1-8 (2000).

7. G.D. Badhwar, W. Atwell, E.V. Benton, A.L. Frank, R.P. Keegan, V.E. Dudkin, O.N. Karpov, Yu.V. Potapov, A.B. Akopova, N.V. Magradze, L.V. Melkumyan and Sh.B. Rshtuni, A study of the radiation environment on board the Space Shuttle flight STS-57. Radiat. Meas. 24(3), 283-289 (1995). 
8 J. A. Simpson, in Composition and Origin of Cosmic Rays, edited by M. M. Shapiro (Reidel, Dordrecht, 1983), p. 1

9. J.W. Wilson, M. Kim, W. Schimmerling, F.F. Badavi, S.A. Thibeault, F.A. Cucinotta, J.L. Shinn and R. Kiefer, Issues in space radiation protection: galactic cosmic rays. Health Phys. 68, 50-58 (1995).

10. J. R. Letaw, R. Silberberg and C. H. Tsao, Radiation hazards on space missions outside the magnetosphere. Adv. Space Res. 9(10), 285-291 (1989).

11. C. Zeitlin, J. Miller, L. Heilbronn, K. Frankel, W. Gong and W. Schimmerling, The fragmentation of $510 \mathrm{MeV} /$ nucleon iron-56 in polyethylene. I. Fragment fluence spectra. Radiat. Res. 145, 655-665 (1996).

12. C. Zeitlin, L. Heilbronn, J. Miller, S. E. Rademacher, T. Borak, T. R. Carter, K. A. Frankel, W. Schimmerling and C. E. Stronach, Heavy fragment production cross sections from 1.05 GeV/nucleon ${ }^{56} \mathrm{Fe}$ in $\mathrm{C}, \mathrm{Al}, \mathrm{Cu}, \mathrm{Pb}$ and $\mathrm{CH}_{2}$ targets. Phys. Rev. C 56, 388-397 (1997).

13. C. Zeitlin, L. Heilbronn, J. Miller, Detailed characterization of the $1087 \mathrm{MeV} /$ nucleon ${ }^{56} \mathrm{Fe}$ beam used for radiobiology at the AGS. Radiat. Res. 149, 560-569 (1998).

14. C. Zeitlin, A. Fukumura, L. Heilbronn, Y. Iwata, J. Miller, T. Murakami, Fragmentation Cross Sections of $600 \mathrm{MeV} /$ nucleon ${ }^{20} \mathrm{Ne}$ on Elemental Targets. Phys. Rev. C 64, 024902 (2001).

15. E.A. Blakely and A. Kronenberg, Heavy ion radiobiology: new approaches to delineate mechanisms underlying enhanced biological effectiveness. Radiat. Res. 150, S126-S145 (1998).

16. S. B. Curtis, J. E. Nealy and J. W. Wilson, Risk cross sections and their application to risk estimation in the galactic cosmic-ray environment. Radiat. Res. 141, 57-65 (1995). 
17. C. Zeitlin. L. Heilbronn, J. Miller, W. Schimmerling, L.W. Townsend, R.K. Tripathi and J.W. Wilson, The fragmentation of $510 \mathrm{MeV} /$ nucleon iron-56 in polyethylene. II. Comparisons between data and a model. Radiat. Res. 145, 666-672 (1996).

18. J. W. Wilson, J. L. Shinn, L. W. Townsend, R. K. Tripathi, F. Badavi, and S. Y. Chun, NUCFRG2: A Semiempirical Nuclear Fragmentation Model. Nucl. Instr. Meth. Phys. Res. B 94, 95-102 (1994). 
Table 1

Composition of ISS hull and crew quarter wall materials

\begin{tabular}{|c|c|c|}
\hline Location & Composition & Thickness \\
\hline \hline ISS Hull & & \\
\hline & & \\
\hline pressure vessel & & $1.39 \mathrm{gm} / \mathrm{cm}^{2}$ \\
\hline debris bumper & $\mathrm{Al} 2219$ alloy & $0.54 \mathrm{gm} / \mathrm{cm}^{2}$ \\
\hline & $\mathrm{Al} 6061$ alloy & \\
\hline Crew Quarter wall & & \\
\hline & & $0.08 \mathrm{gm} / \mathrm{cm}^{2}$ \\
\hline Nomex cloth & & $0.06 \mathrm{gm} / \mathrm{cm}^{2}$ \\
\hline Durette batting & $\mathrm{H}, \mathrm{C}, \mathrm{N}, \mathrm{O}$ & $0.72 \mathrm{gm} / \mathrm{cm}^{2}$ \\
\hline silicone rubber & $\mathrm{H}, \mathrm{C}, \mathrm{N}, \mathrm{O}$ & $0.22 \mathrm{gm} / \mathrm{cm}^{2}$ \\
\hline Nomex honeycomb & $\mathrm{SiO}, \mathrm{CH}$ & \\
\hline
\end{tabular}

Table 2

Track-average LET $(\mathrm{keV} / \mu \mathrm{m})$ for the target material samples as measured in each of three detector pairs downstream of the target location.

\begin{tabular}{|l|c|c|c|}
\hline \multicolumn{1}{|c|}{ Target } & $\langle\mathrm{L}\rangle_{\mathrm{PSD} 2}$ & $\langle\mathrm{~L}\rangle_{\mathrm{d} 3 \mathrm{~mm} 1 / 2}$ & $\langle\mathrm{~L}\rangle_{\mathrm{d} 3 \mathrm{~mm} 3 / 4}$ \\
\hline \hline ISS Hull & 142 & 141 & 140 \\
\hline Hull + CQ Wall & 138 & 137 & 135 \\
\hline Hull + encapsulated PE & 132 & 129 & 127 \\
\hline Hull + PE-augmented CQ Wall & 130 & 126 & 123 \\
\hline Hull + PE-augmented CQ Wall + encapsulated PE & 120 & 114 & 109 \\
\hline
\end{tabular}




\section{Table 3}

Ratios of the track-average LET, ICRP60 quality factor and the product of the two for each target, measured in the detector pair furthest from the target to the values for aluminum ISS hull alone.

\begin{tabular}{|l|c|c|c|}
\hline \multicolumn{1}{|c|}{ Target } & $\langle\mathrm{L}\rangle_{\text {trk }}$ & $\langle\mathrm{Q}\rangle_{\text {trk }}$ & $\langle\mathrm{L} \cdot \mathrm{Q}\rangle_{\text {trk }}$ \\
\hline \hline Hull + CQ Wall & .96 & .98 & .97 \\
\hline Hull + encapsulated PE & .90 & .94 & .90. \\
\hline Hull + PE-augmented CQ Wall & .87 & .93 & .87. \\
\hline Hull + PE-augmented CQ Wall + encapsulated PE & .78 & .86 & .77. \\
\hline
\end{tabular}

Notes. The values for the $7 \mathrm{~mm}$ aluminum hull are: $\langle\mathrm{L}\rangle_{\mathrm{trk}}=140 \mathrm{keV} / \mu \mathrm{m},\langle\mathrm{Q}\rangle_{\mathrm{trk}}=23.74,\langle\mathrm{~L} \cdot \mathrm{Q}\rangle_{\mathrm{trk}}=3452$.

\section{Table 4}

Comparison of measured and calculated dose- and track-average LET (keV/ $/ \mu \mathrm{m})$ and ICRP60 quality factor behind ISS hull material $(0.7 \mathrm{~cm} \mathrm{Al})$ with polyethylene augmentation $(0.1 \mathrm{~cm}$ graphite-epoxy $+1.2 \mathrm{~cm}$ polyethylene $+0.1 \mathrm{~cm}$ graphite-epoxy.

\begin{tabular}{|l|c|c|c|c|c|}
\cline { 2 - 6 } \multicolumn{1}{c|}{} & $\begin{array}{c}1000 \mathrm{MeV} / \mathrm{nuc} \\
\text { (Data) }\end{array}$ & $\begin{array}{c}1000 \mathrm{MeV} / \mathrm{nuc} \\
(\mathrm{Model})\end{array}$ & $\begin{array}{c}600 \mathrm{MeV} / \mathrm{nuc} \\
(\mathrm{Model})\end{array}$ & $\begin{array}{c}400 \mathrm{MeV} / \mathrm{nuc} \\
\text { (Model) }\end{array}$ & $\begin{array}{c}300 \mathrm{MeV} / \mathrm{nuc} \\
\text { (Model) }\end{array}$ \\
\hline$\langle\mathrm{L}\rangle_{\text {trk }}$ & 132 & 133 & 164 & 217 & 333 \\
\hline$\langle\mathrm{Q}\rangle_{\text {trk }}$ & 23 & 23 & 21 & 18 & 16 \\
\hline$\langle\mathrm{L}\rangle_{\text {dose }}$ & 143 & 147 & 178 & 237 & 358 \\
\hline $\begin{array}{l}\langle\mathrm{Q}\rangle \\
\text { dose }\end{array}$ & 25 & 24 & 22 & 19 & 16 \\
\hline
\end{tabular}


Table 5

Calculated track-average LET $(\mathrm{keV} / \mu \mathrm{m})$ for aluminum and aluminum augmented with polyethylene for iron ions at four different incident energies

\begin{tabular}{|c|c|c|c|c|}
\cline { 2 - 5 } \multicolumn{1}{c|}{} & $\begin{array}{c}1000 \mathrm{MeV} / \mathrm{nuc} \\
(\text { Model })\end{array}$ & $\begin{array}{c}600 \mathrm{MeV} / \mathrm{nuc} \\
(\mathrm{Model})\end{array}$ & $\begin{array}{c}400 \mathrm{MeV} / \mathrm{nuc} \\
\text { (Model) }\end{array}$ & $\begin{array}{c}300 \mathrm{MeV} / \mathrm{nuc} \\
\text { (Model) }\end{array}$ \\
\hline $0.7 \mathrm{~cm} \mathrm{Al}$ & 150 & 173 & 205 & 238 \\
\hline $\begin{array}{l}\text { no material } \\
0.7 \mathrm{~cm} \text { Al }+1.2 \mathrm{~cm} \text { poly } \\
+0.2 \mathrm{~cm} \text { graphite-epoxy }\end{array}$ & 133 & 167 & 207 & 259 \\
\hline
\end{tabular}




\section{Figure Captions}

FIG. 1. Detector configuration (not to scale). All detectors are lithium-drifted silicon. The respective thicknesses and approximate active areas are: TR - $300 \mu \mathrm{m} \times 300 \mathrm{~mm} 2$; PSD1 and PSD2 - 1mm x $1500 \mathrm{~mm}^{2}$; d3mmU and d3mm1-4 - 3mm x $450 \mathrm{~mm}^{2}$.

FIG. 2. Summed energy loss $(\mathrm{MeV})$ in the detector pair $\mathrm{d} 3 \mathrm{~mm} 3 / 4$ for primary ions and fragments produced by $1037 \mathrm{MeV} / \mathrm{u}^{56} \mathrm{Fe}$ ions interacting in ISS hull material ( $7 \mathrm{~mm}$ aluminum). The ordinate is the number of particles in the detector pair which satisfy the selection criteria.

FIG. 3. Spectra of primary ions and fragment charges produced by $1037 \mathrm{MeV} / \mathrm{u}{ }^{56} \mathrm{Fe}$ ions interacting in the ISS hull. These spectra were obtained by scaling the summed energy loss in the detector pairs

(a) PSD2X+PSD2Y, (b) $\mathrm{d} 3 \mathrm{~mm} 1+\mathrm{d} 3 \mathrm{~mm} 2$ and (c) $\mathrm{d} 3 \mathrm{~mm} 3+\mathrm{d} 3 \mathrm{~mm} 4$ as described in the text.

(d) Spectrum in Fig. $3 \mathrm{~b}$ with signals amplified to increase the sensitivity to small charges.

(Figure 3c corresponds to the energy loss spectrum in Fig. 2.)

FIG. 4. Fragment fluence, normalized to a single beam ion, for each material combination as a function of fragment charge measured in the detector pair furthest downstream $(\mathrm{d} 3 \mathrm{~mm} 3 / 4)$. The errors are statistical. Error bars not shown are smaller than the data points.

FIG. 5. Fluence in Fig. 4 as a function of fragment LET. The abscissa value for each point is the $\mathrm{LET}_{\infty}$ for the corresponding fragment charge, assuming that the fragment is at the beam velocity. The solid lines are to guide the eye.

FIG. 6. Product of fluence and LET ${ }_{\infty}$ for each fragment charge state, for the data in Figs. 4 and 5.

FIG. 7. Product of fluence and LET ${ }_{\infty}$, weighted by the ICRP60 quality factor for that LET (inset).

FIG. 8. Fraction of primary ions, measured in $\mathrm{d} 3 \mathrm{~mm} 3 / 4$, remaining after passing through each target, plotted vs. the track-average LET, defined as the product of fluence and LET (assuming beam velocity) for each measured charge, summed over all measured charges.

FIG. 9 (a) Calculated track-average LET for iron ions at energies between 200 and 1000 $\mathrm{MeV} /$ nucleon fragmenting in the $0.7 \mathrm{~cm}$ aluminum ISS hull augmented with $0,1,2,5$ and $10 \mathrm{~cm}$ polyethylene. (b) Calculated track-average LET weighted by the ICRP60 quality factor Q(LET).

FIG. 10 Same as Fig. 9, for silicon ions. 


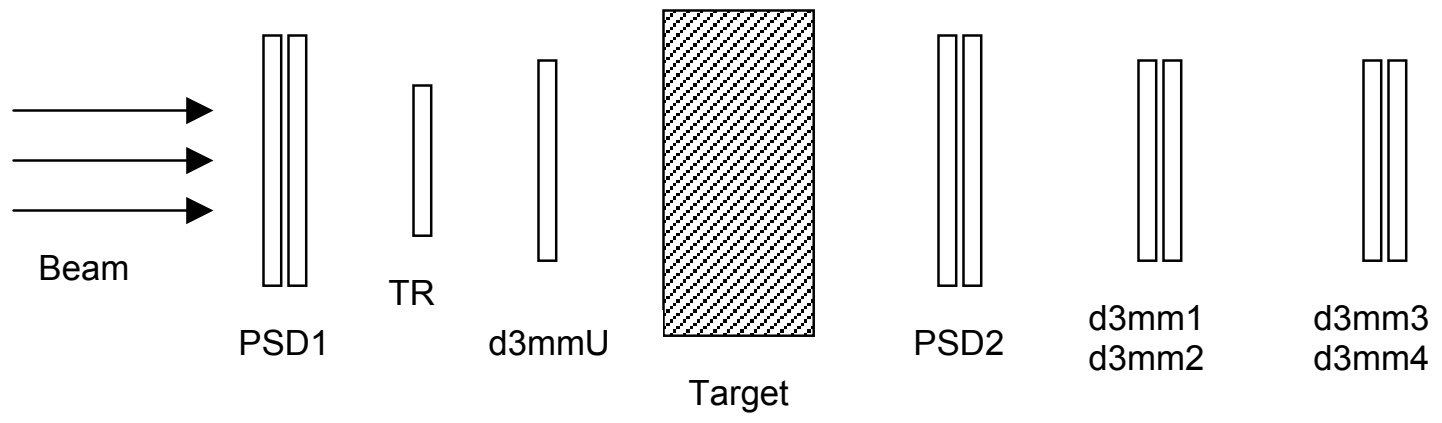




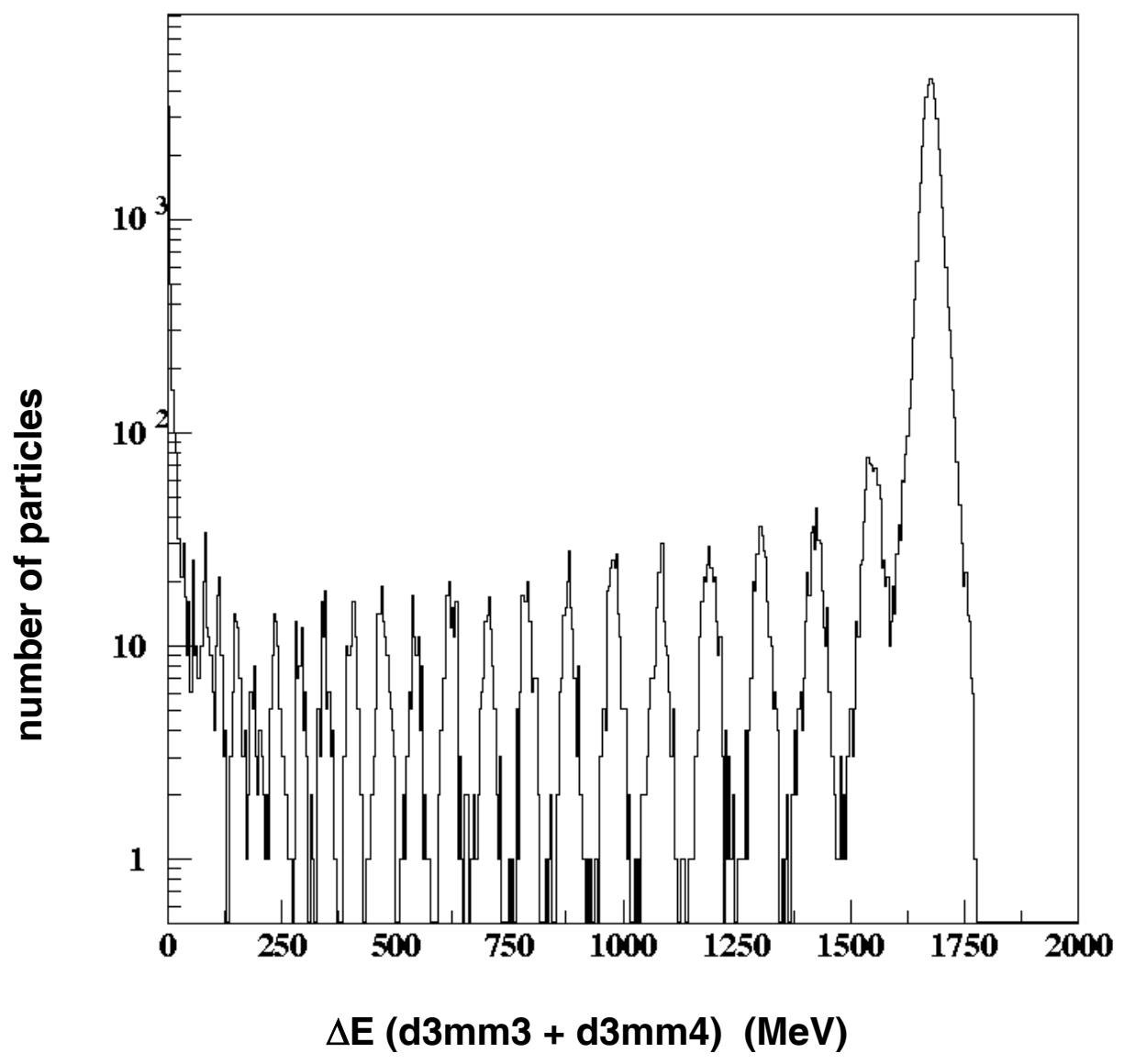



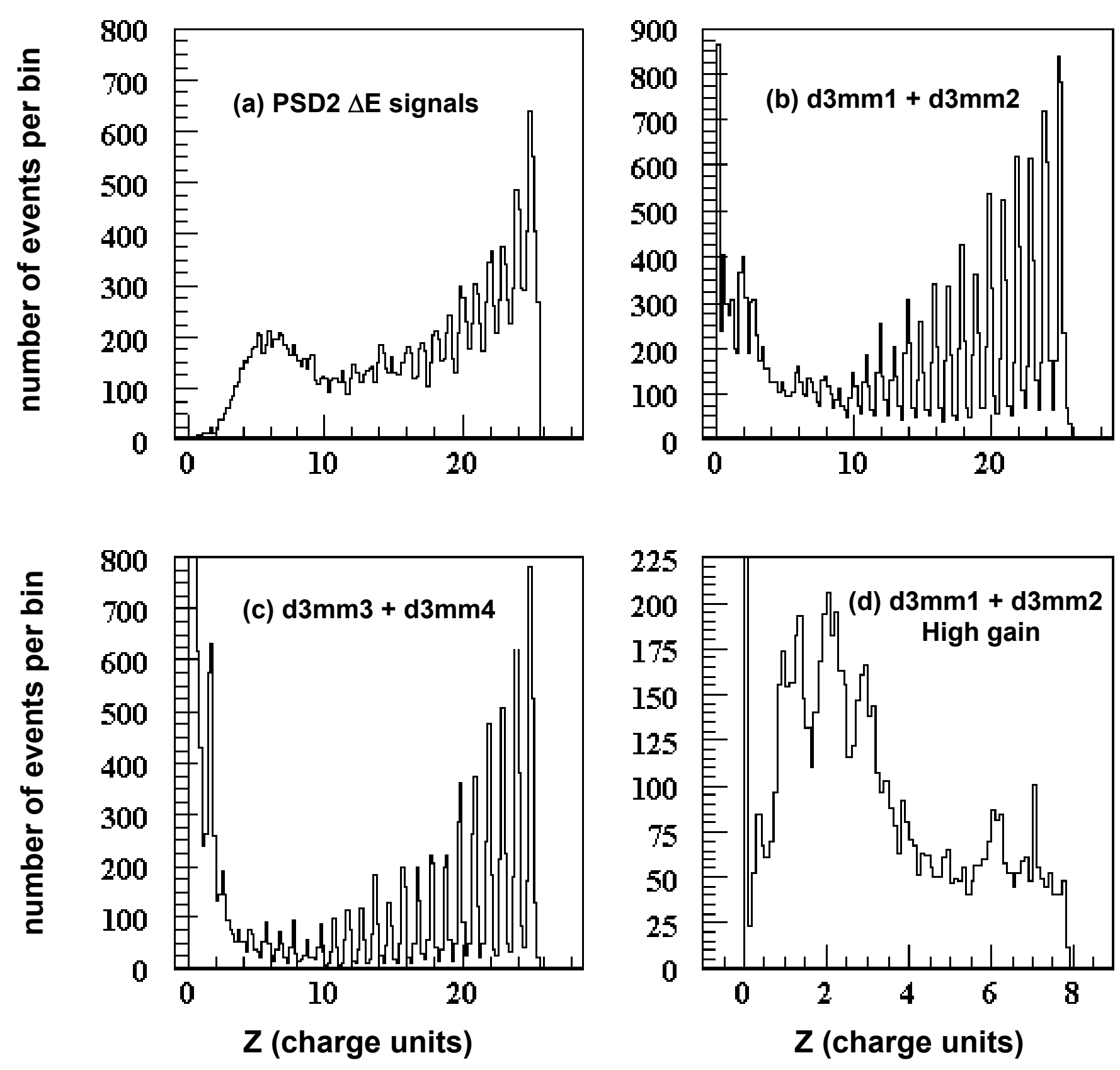


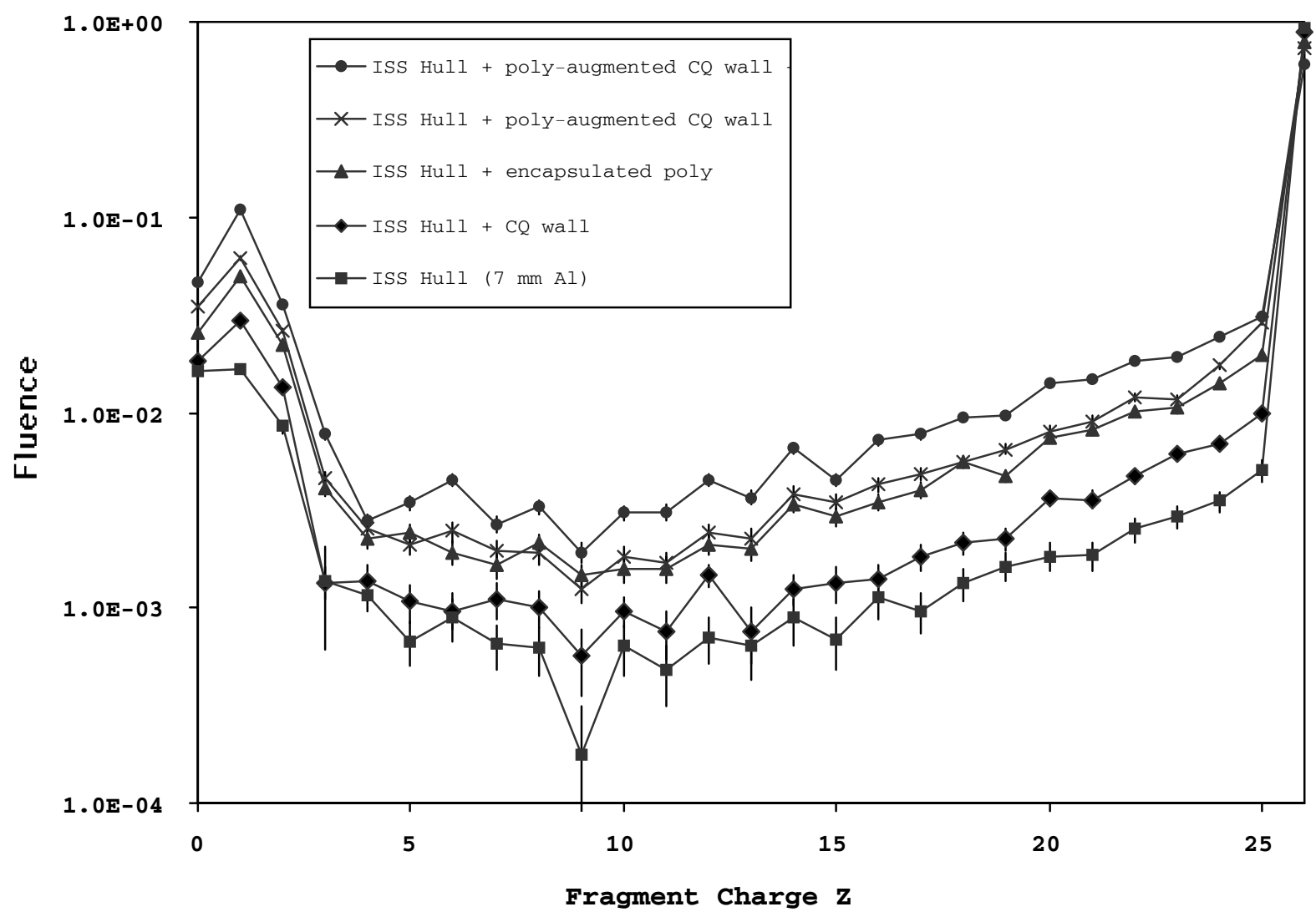




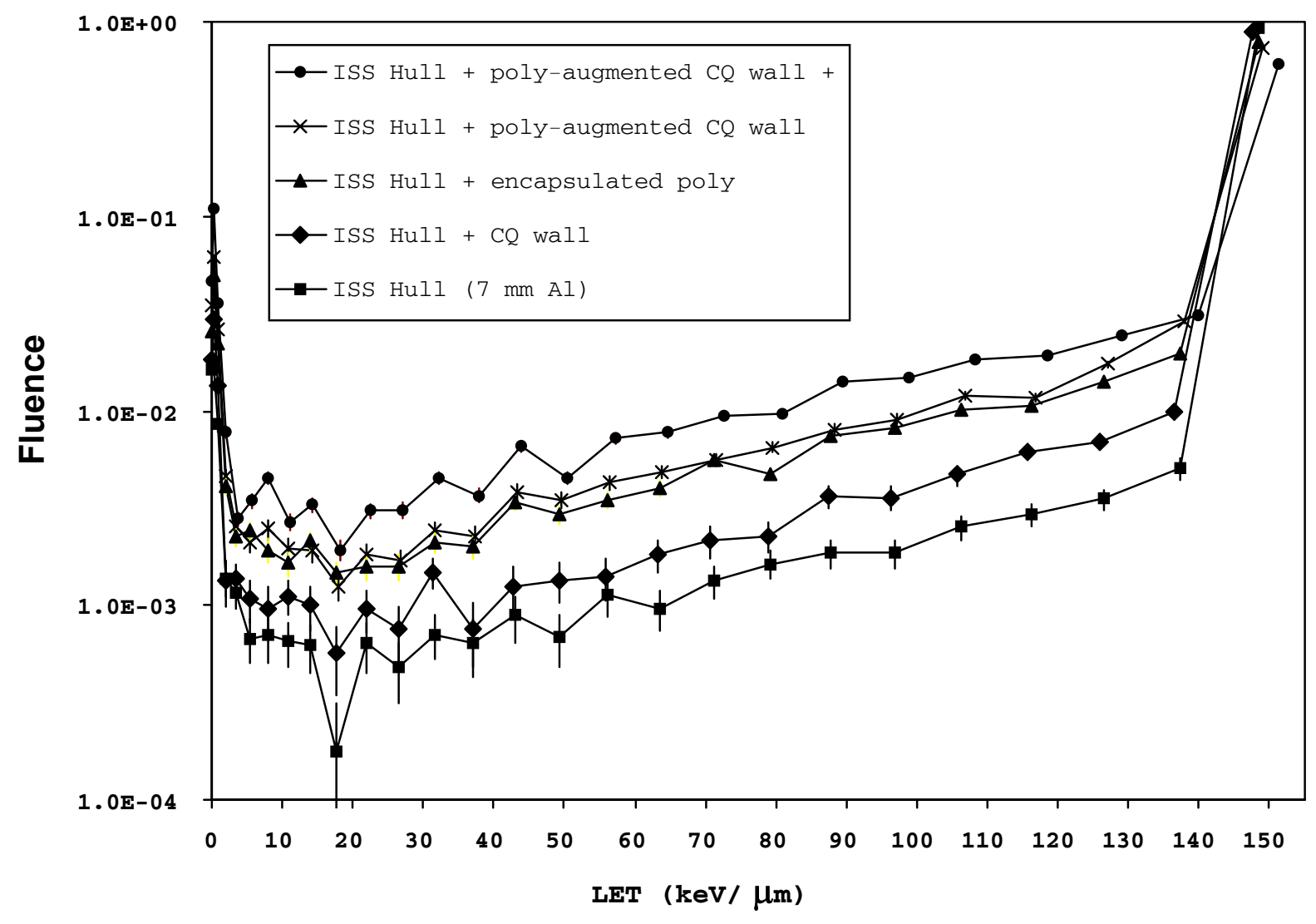




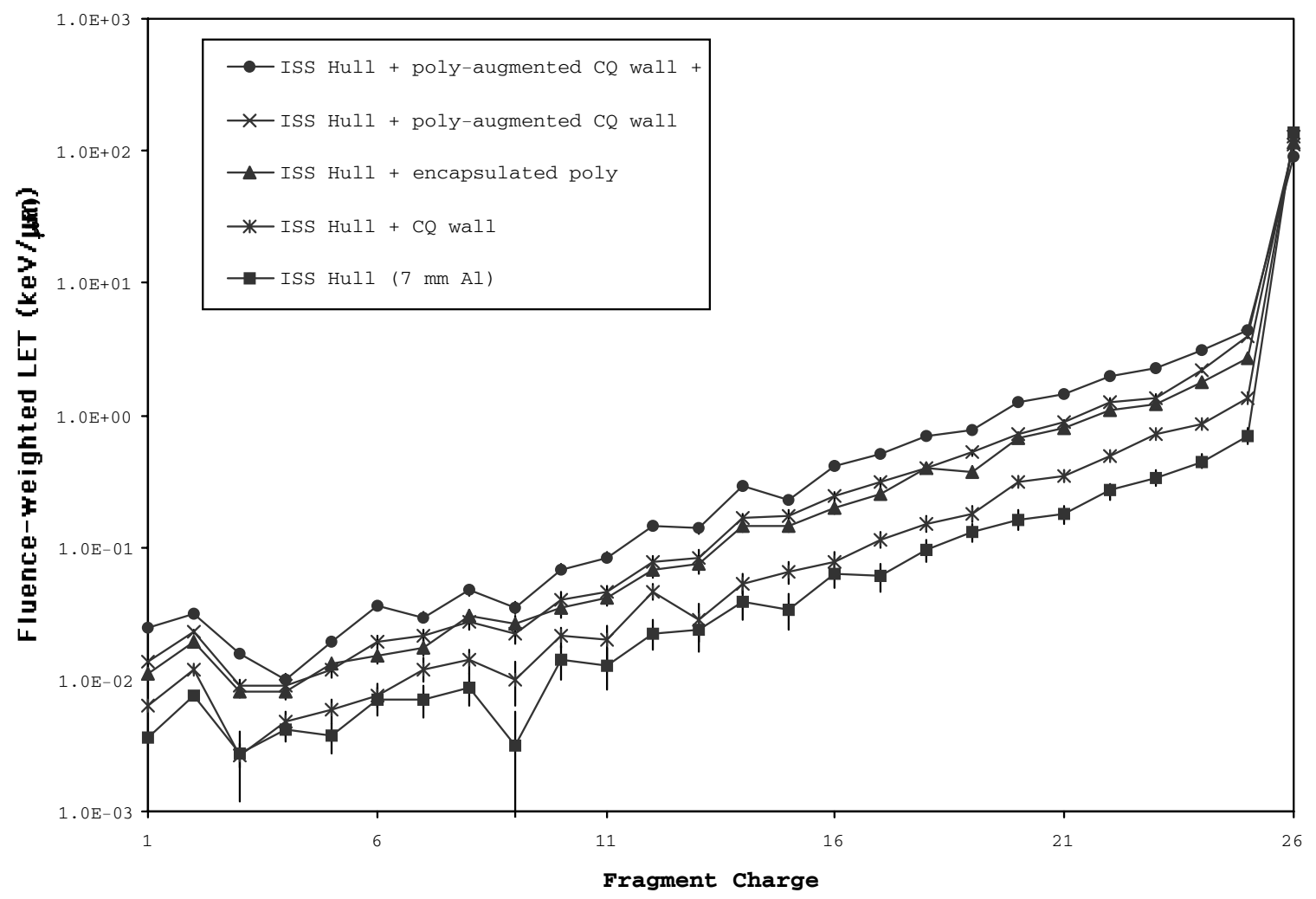




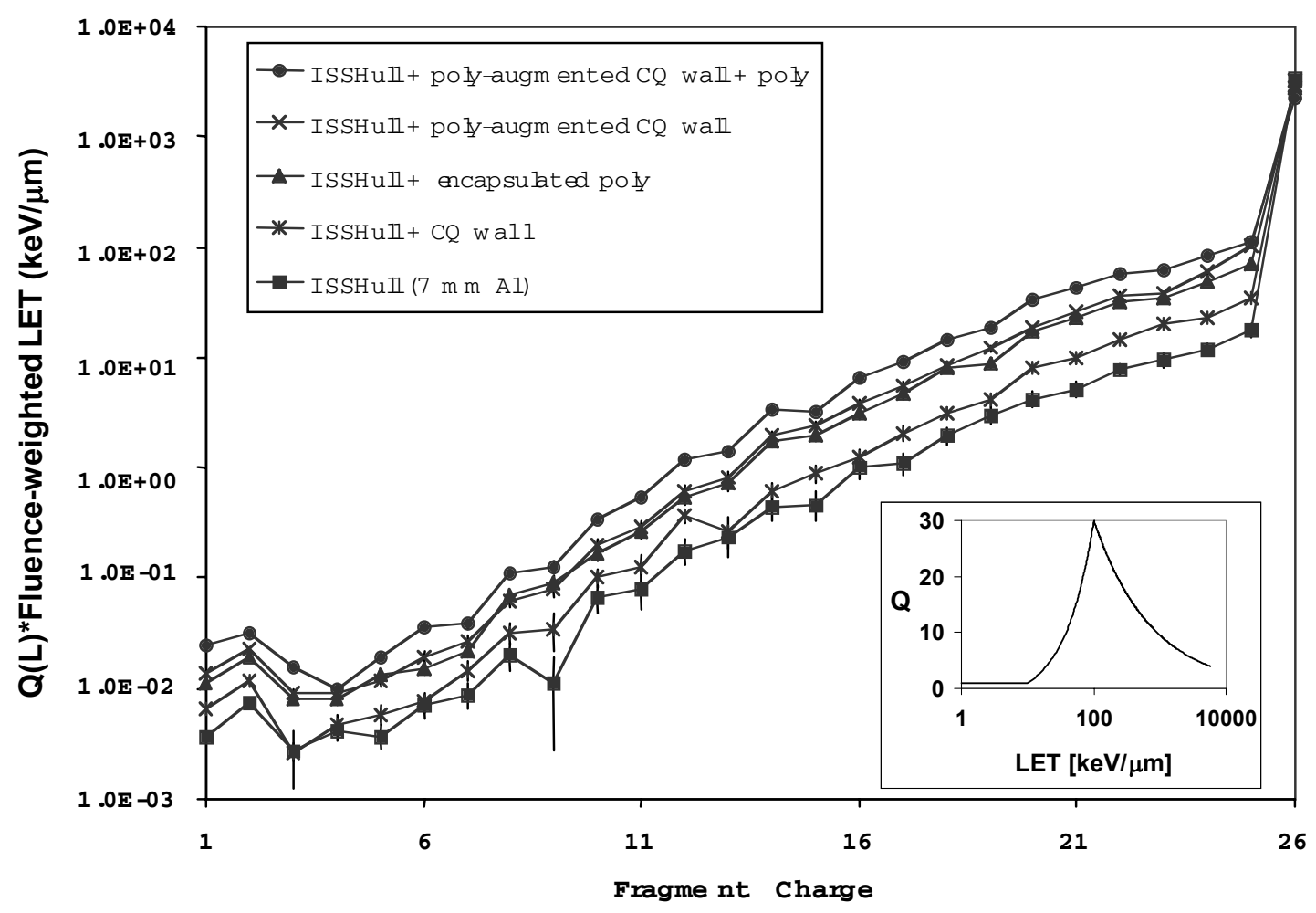




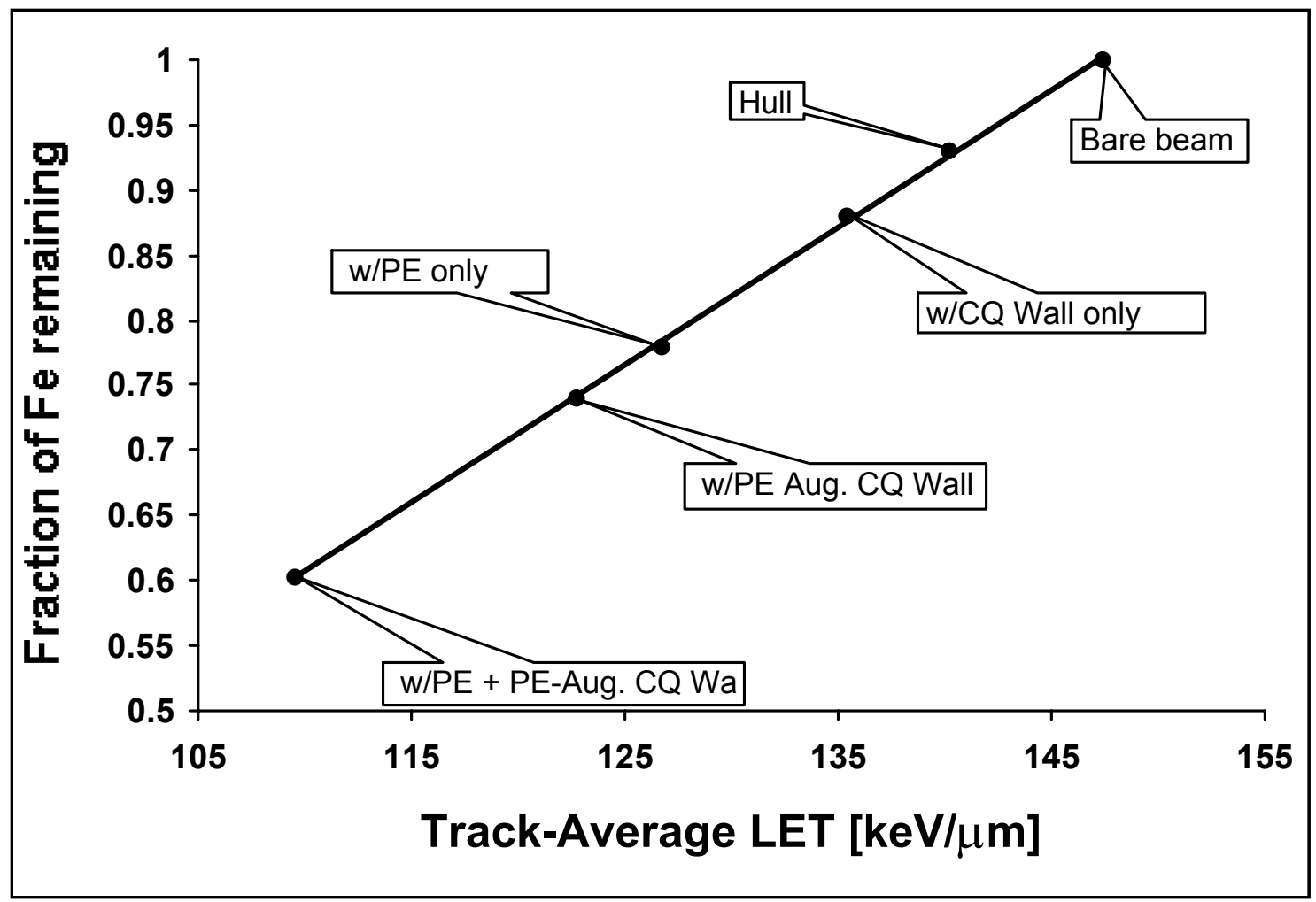



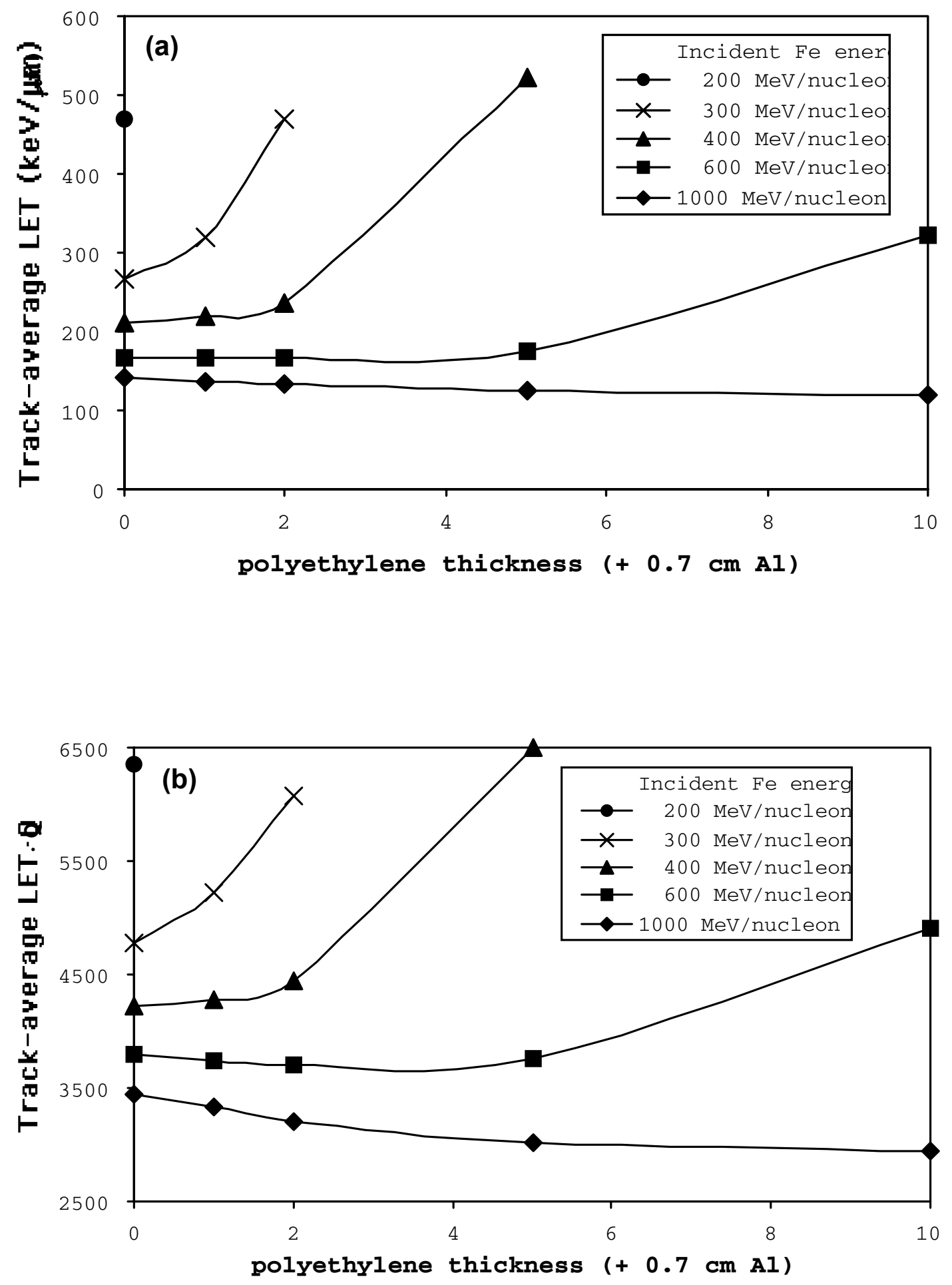

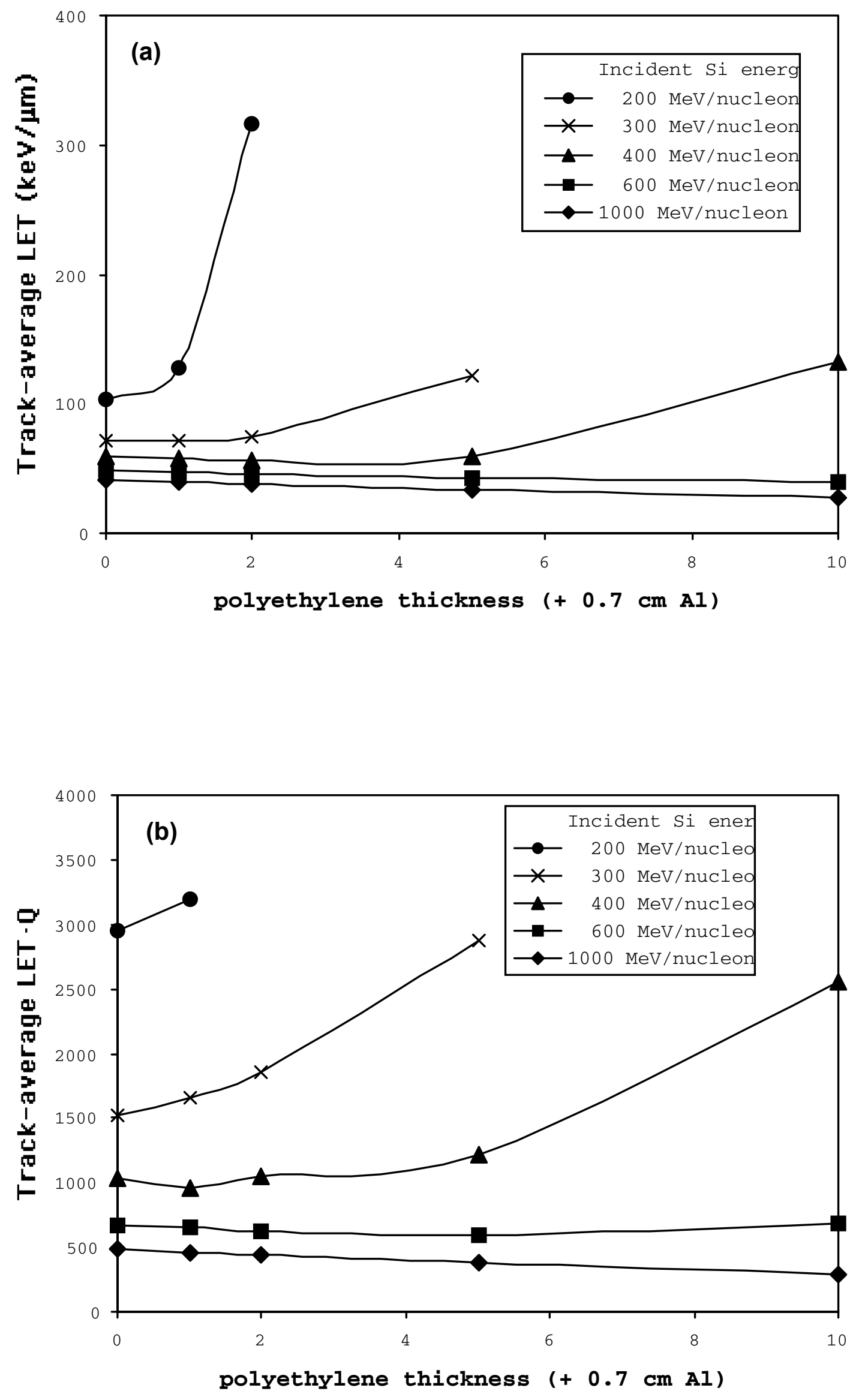